\title{
Diffraction by gratings with random fill factor
}

\author{
Francisco Jose Torcal-Milla ${ }^{1, *}$ AND LUIS Miguel Sanchez-BreA ${ }^{2}$ \\ ${ }^{1}$ Departamento de Física Aplicada, Universidad de Zaragoza, 50009, Zaragoza (Spain) \\ ${ }^{2}$ Applied Optics Complutense Group, Optics Department, Facultad de Ciencias Físicas, Plaza de las Ciencias, 1, Universidad Complutense de Madrid, 28040, \\ Madrid (Spain) \\ *Corresponding author: fjtorcal@unizar.es
}

Compiled May 11, 2017

\begin{abstract}
In this work, we analyze the diffraction produced by Ronchi gratings where the fill factor is not constant, but it presents random fluctuations around its nominal value. This effect can be produced during developing the grating with etchers since the process can be slightly unpredictable. We obtain the theoretical formalism to describe the intensity produced by the grating at near and far field showing that smoothing of the self-images is produced at the near field and, consequently, cancellation of higher diffraction orders is obtained at the far field. In addition, different nominal fill factors produce different diffraction behavior in terms of the randomness. We corroborate the analytical formalism by using a direct integration method based on the Rayleigh-Sommerfeld formula concluding that the numerical results are in high agreement with the theoretical predictions. () 2017 Optical Society of America
\end{abstract}

OCIS codes: (050.0050) Diffraction and gratings; (050.1950) Diffraction gratings;(030.0030) Coherence and statistical optics.

http://dx.doi.org/10.1364/ao.XX.XXXXXX

\section{INTRODUCTION}

One of the most used optical elements is the diffraction grating. It consists of a periodical pattern that modulates the incident light. Diffraction gratings are useful elements in many different branches of science and applications such as metrology, laser array illumination, moiré interferometry, spectroscopy, phase locking of laser arrays, etc, [1-4]. Particularly, in optics, the most common kinds of diffraction gratings are amplitude-based and phase-based gratings that modulate the amplitude and the phase of the incident beam respectively, [5], but other types of gratings such as polarization gratings or rough gratings have been introduced in recent years. Rough gratings can be understood as a modulation of the coherence state of the beam, [6-8]. Considering the shape or transmittance of the grating, diffraction gratings with fill factor being half of the period are more common but other relationships between period and fill factor are possible, [9].

Concerning fabrication methods, diffraction gratings are commonly manufactured by photo-lithographic methods, direct laser writing, or laser ablation. The most adequate method for fabrication depends on the substrate of the grating, the desired grating type, the needed accuracy, the desired feature, and so on. For example, gratings over glass substrates are usually fabricated by photo-lithographic methods [10] and gratings over steel substrates are commonly manufactured by laser ablation [11]. Despite manufacturing processes have been improved year after year, manufacturing errors are still present and become more notorius when period of the gratings approaches the wavelength of the incident light beam. The effect of different kinds of imperfections in diffraction gratings such as rough edges of the slits [12], random distribution of phase delays [13], roughness on the surface, [14-16], missing slits, [17], random distributions [18], random positioning of the slits forming the grating [19], etc, have been analyzed in recent years. In this manuscript, we analytically and numerically investigate the near and far field diffraction pattern of Ronchi gratings formed by an ensemble of transparent slits with random fill factor. As it has been introduced previously, diffraction gratings behavior has been studied from many points of view. Particularly, it is well known that Talbot effect is produced at the near field, consisting of the replication of the grating pattern at different distances from the grating, called Talbot distances, [20-22]. The Talbot distance is defined as $z_{T}=2 p^{2} / \lambda$, where $p$ is the period of the grating and $\lambda$ is the illumination wavelength. In our case, as we demonstrate following, the near field intensity produced by the proposed grating is slightly different. Randomness affects to the smoothness of the self-images of the grating, decreasing the contrast in terms of the randomness level and producing the disappearance of the self-images for high randomness. Consequently, cancellation of high diffraction orders at the far field is also produced. Numerical simulations made by means of a numerical integration of the Rayleigh-Sommerfeld formula corroborate the analytical results. 


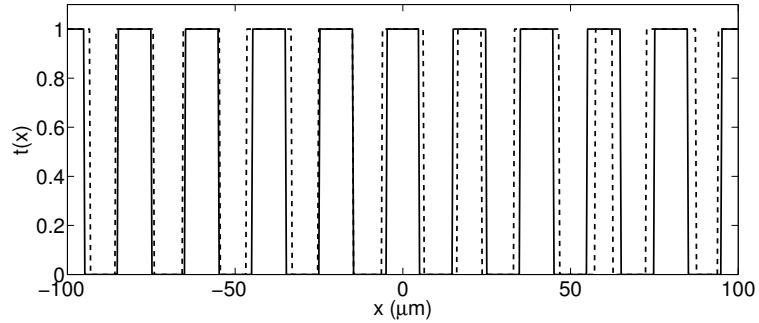

Fig. 1. Example of amplitude diffraction grating proposed, $\omega=p / 10$, (dashed line) versus periodical grating with constant fill factor, $\tau=p / 2$, (solid line). The period of the grating is $p=20 \mu m$.

\section{NEAR-FIELD APPROACH}

Let us consider a one-dimensional diffraction grating whose transmittance is defined as the summation of transparent slits with random fill factor. It can be mathematically expressed as

$$
t_{r}\left(x^{\prime}\right)=\sum_{n} \operatorname{rect}\left(\frac{x^{\prime}-n p}{\tau+\alpha_{n}}\right),
$$

where $n$ are integer numbers, $p$ is the period of the grating, $\tau$ is the nominal fill factor of the grating, and $\alpha_{n}$ is a random number following a certain probabilistic distribution. We show in Figure 1 an example of the grating proposed (dashed line) and a periodical grating of the same period with constant fill factor, $\tau=p / 2$, (solid line). In the figure caption, $\omega$ defines the width of the distribution of random $\alpha_{n}$, considering gaussian, as we show in the following section.

Considering non-polarized monochromatic plane wave illumination, the diffracted pattern at the near field is calculated by using the Fresnel approach as

$$
U(x, z)=A \frac{\exp (i k z)}{\sqrt{i k z}} \int_{-\infty}^{+\infty} t_{r}\left(x^{\prime}\right) \exp \left[\frac{i \pi}{\lambda z}\left(x-x^{\prime}\right)^{2}\right] d x^{\prime},
$$

where $A$ is the amplitude of the incident field, $z$ is the distance from the grating to the observation plane, $\lambda$ is the wavelength of the incoming wave, and $x$ is the transversal coordinate at the observation plane. Applying Eq. (1) to Eq. (2), the field can be rewritten as

$$
U(x, z)=A \frac{\exp (i k z)}{\sqrt{i k z}} \sum_{n} \int_{n p-\left(\tau+\alpha_{n}\right) / 2}^{n p+\left(\tau+\alpha_{n}\right) / 2} \exp \left[\frac{i \pi}{\lambda z}\left(x-x^{\prime}\right)^{2}\right] d x^{\prime} .
$$

The integral is easily solved resulting

$$
U\left(x, z, \alpha_{n}\right)=-\frac{1}{2} A \exp (i k z) \sum_{n}\left[\operatorname{erf}\left(\varepsilon_{n}+\mu \alpha_{n}\right)+\operatorname{erf}\left(\theta_{n}+\mu \alpha_{n}\right)\right],
$$

where

$$
\begin{aligned}
\operatorname{erf}(\chi) & =2 / \sqrt{\pi} \int_{0}^{\chi} \exp \left(-t^{2}\right) d t, \\
\varepsilon_{n} & =-(1+i)(n p-x+\tau / 2) \sqrt{\pi /(2 z \lambda)}, \\
\theta_{n} & =-(1+i)(-n p+x+\tau / 2) \sqrt{\pi /(2 z \lambda),} \\
\mu & =-(1+i) \sqrt{\pi /(8 z \lambda)} .
\end{aligned}
$$

The random character of the diffraction grating forces to use an averaging process to calculate the intensity distribution at the near field. This averaging process can be understood as a longitudinal displacement of the grating, as it occurs in linear optical encoders, [23, 24], or as a result of the manufacturing process. To obtain analytical results, we have chosen the random probabilistic function as gaussian with null mean value

$$
p\left(\alpha_{j}\right)=\frac{1}{\sqrt{2 \pi} \omega} \exp \left(-\frac{\alpha_{j}^{2}}{2 \omega^{2}}\right) ; \quad j=n, m,
$$

where $\omega$ is the standard deviation of the random fill factor with respect to the nominal value [25]. Then, the average intensity can be calculated as

$$
\begin{aligned}
<I(x, z)> & =<U\left(x, z, \alpha_{n}\right) U^{*}\left(x, z, \alpha_{m}\right)> \\
& =\int_{-\infty}^{+\infty} \int_{-\infty}^{+\infty} p\left(\alpha_{n}, \alpha_{m}\right) U\left(x, z, \alpha_{n}\right) U^{*}\left(x, z, \alpha_{m}\right) d \alpha_{n} d \alpha_{m}
\end{aligned}
$$

where $p\left(\alpha_{n}, \alpha_{m}\right)$ is the joint probabilistic function of both distributions, [25]. Considering that the random variables are uncorrelated, $p\left(\alpha_{n}, \alpha_{m}\right)=p\left(\alpha_{n}\right) p\left(\alpha_{m}\right)$, the average intensity can be rewritten as

$$
\begin{aligned}
<I(x, z)>= & \int_{-\infty}^{+\infty} p\left(\alpha_{n}\right) U\left(x, z, \alpha_{n}\right) d \alpha_{n} \\
& \int_{-\infty}^{+\infty} p\left(\alpha_{m}\right) U^{*}\left(x, z, \alpha_{m}\right) d \alpha_{m} .
\end{aligned}
$$

Placing all terms into Eq. (7) and solving the integrals, the average intensity results

$$
<I(x, z)>=\left|\frac{A}{2} \sum_{n}\left[\operatorname{erf}\left(\frac{\varepsilon_{n}}{1+2 \mu^{2} \omega^{2}}\right)+\operatorname{erf}\left(\frac{\theta_{n}}{1+2 \mu^{2} \omega^{2}}\right)\right]\right|^{2} .
$$

Substituting variables and simplifying, the average intensity can be expressed as

$$
\begin{gathered}
<\quad I(x, z)>=\frac{A}{2} \mid \sum_{n}\left\{\operatorname{erf}\left[\sqrt{\frac{2 \pi}{\pi \omega^{2}-2 i z \lambda}}\left(n p-x-\frac{\tau}{2}\right)\right]\right. \\
\left.+\operatorname{erf}\left[-\sqrt{\frac{2 \pi}{\pi \omega^{2}-2 i z \lambda}}\left(n p-x+\frac{\tau}{2}\right)\right]\right\}\left.\right|^{2} .
\end{gathered}
$$

It corresponds to the near field diffraction pattern produced by a Ronchi grating with fill factor half of the period but corrected by the term $\pi \omega^{2}$, due to randomness. As it would be expected, we recover the solution without randomness considering $\omega \rightarrow$ 0 . Following, we show in Figure 2 four examples of average intensity at the near field calculated by using Eq. (9).

As can be observed, the self-images become smoother for higher randomness but maintaining the period equal to the grating with nominal values. Besides, the contrast of the self-images decreases in terms of the randomness. This fact is shown in Figure 3, where we show the contrast of the first observable self-image in terms of the randomness. It can be observed that contrast decreases but the self-images are still measurable for randomness around half the period of the grating. Usually, manufacture errors are lesser but we extend the analysis to higher randomness for completeness. The solid line in Figure 3 corresponds with the contrast following the definition given in [26]

$$
C(\omega)=\frac{<I_{\max }>-<I_{\min }>}{<I_{\max }>+<I_{\min }>},
$$

where $<I_{\max }>=\int_{-\infty}^{+\infty} g(x)<I\left(x, z_{T}\right)>d x,<I_{\min }>=$ $\int_{-\infty}^{+\infty}[1-g(x)]<I\left(x, z_{T}\right)>d x$, and $g(x)$ is the grating transmittance without considering randomness. Besides, we show as 


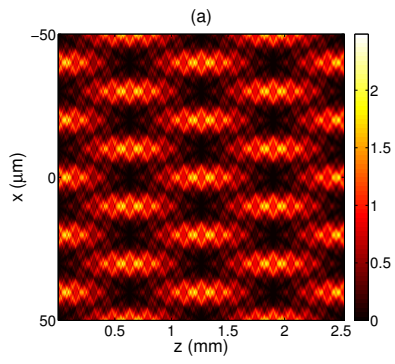

(c)
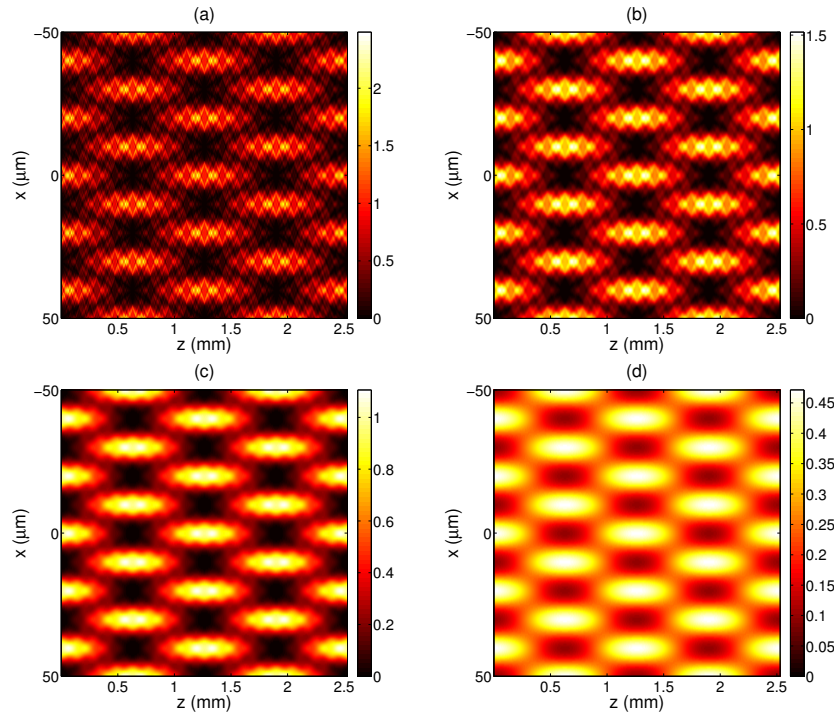

(d)

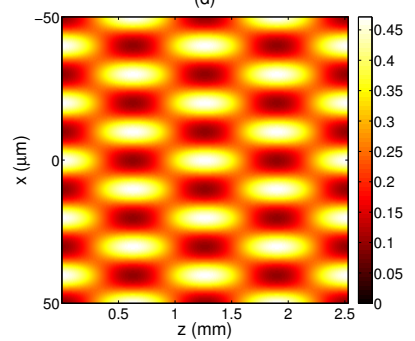

Fig. 2. Analytical self-images calculated using Eq. (9) with a Ronchi grating of period, $p=20 \mu \mathrm{m}$, nominal fill factor $\tau=$ $p / 2$, and illuminated with a plane wave of wavelength, $\lambda=$ $632.8 \mathrm{~nm}$. Colormap corresponds to intensity. The amounts of randomness in the fill factor are: (a) $w=0$, (b) $w=p / 10$, (c) $w=p / 5$, and (d) $w=p / 2$.

dashed line the contrast calculated as

$$
C^{\prime}(\omega)=\left(I_{\text {max }}^{\prime}-I_{\text {min }}^{\prime}\right) /\left(I_{\text {max }}^{\prime}+I_{\text {min }}^{\prime}\right),
$$

where $I_{\text {max }}^{\prime}$ is the maximum average intensity and $I_{\min }^{\prime}$ is the minimum average intensity. The contrast decreases and reaches null contrast for $\omega \approx p$. We have tested this trend and it is independent on the period of the grating and the wavelength.

Let us analyze now the effect of the nominal fill factor on the self-images and its contrast in terms of the randomness. We show in Figure 4 four examples of average intensity calculated by using Eq. (9) with fill factor $\tau=0.2 p$ and different amounts of randomness. In addition, we show in Figure 5 the contrast dependence of the self-images in terms of the randomness for different values of fill factor. In conclusion, smaller fill factor, that is smaller dark slits in our case, results in higher contrast for higher randomness.

\section{FAR-FIELD APPROACH}

To determine the far field diffraction pattern of the grating depicted in Figure 1 (dashed line), we consider the Fraunhofer diffraction kernel for propagation of the light to the far field,

$$
U_{F}(x, z) \propto A \sum_{n} \int_{n p-\left(\tau+\alpha_{n}\right) / 2}^{n p+\left(\tau+\alpha_{n}\right) / 2} \exp \left(\frac{2 i \pi}{\lambda z} x x^{\prime}\right) d x^{\prime},
$$

where we have included the random fill factor in the integration limits.The average intensity is calculated in the same fashion as for near field approach resulting in

$$
I_{F}(x, z) \propto A^{2} \tau^{2} \operatorname{sinc}^{2}\left(\frac{\pi \tau \sin (\theta)}{\lambda}\right) e^{-\left(\frac{k \sin (\theta)}{2}\right)^{2}} \sum_{n, n^{\prime}} e^{i k p\left(n-n^{\prime}\right) \sin (\theta)},
$$

where $\operatorname{sinc}(\phi)=\sin (\phi) / \phi$, and $\sin (\theta)=x / z$. It corresponds to the classical diffraction of a periodic grating but corrected by a term due to the randomness. We show in Figure 6 the

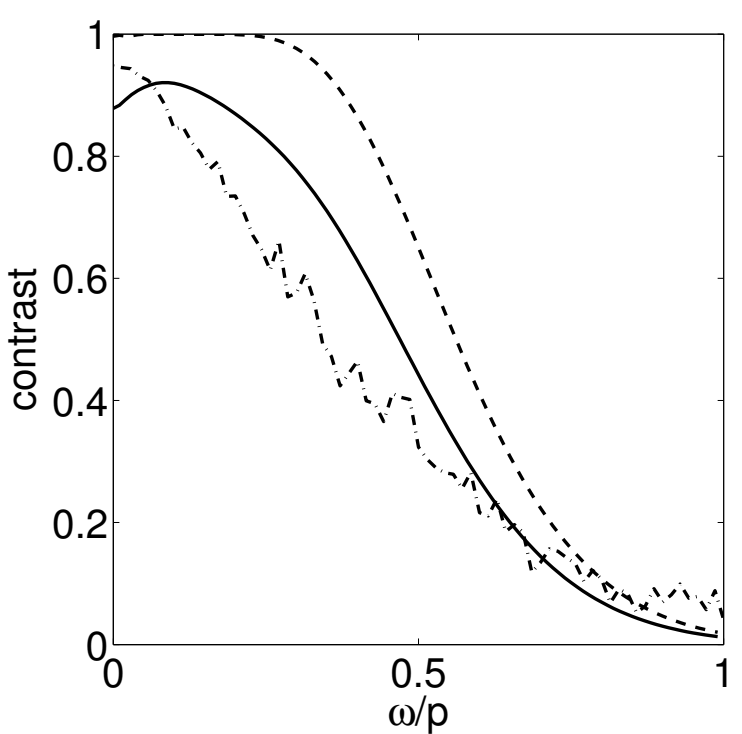

Fig. 3. Contrast of the first observable self-image in terms of randomness in the fill factor. The period of the grating is $p=20 \mu \mathrm{m}$ and the nominal fill factor is $\tau=p / 2$. Analytical calculated by using Eq. (10) (solid line), analytical calculated by using the classical definition of contrast, Eq. (11), (dashed line), and numerically calculated by using the Rayleigh-Sommerfeld approach (dash-dotted line).
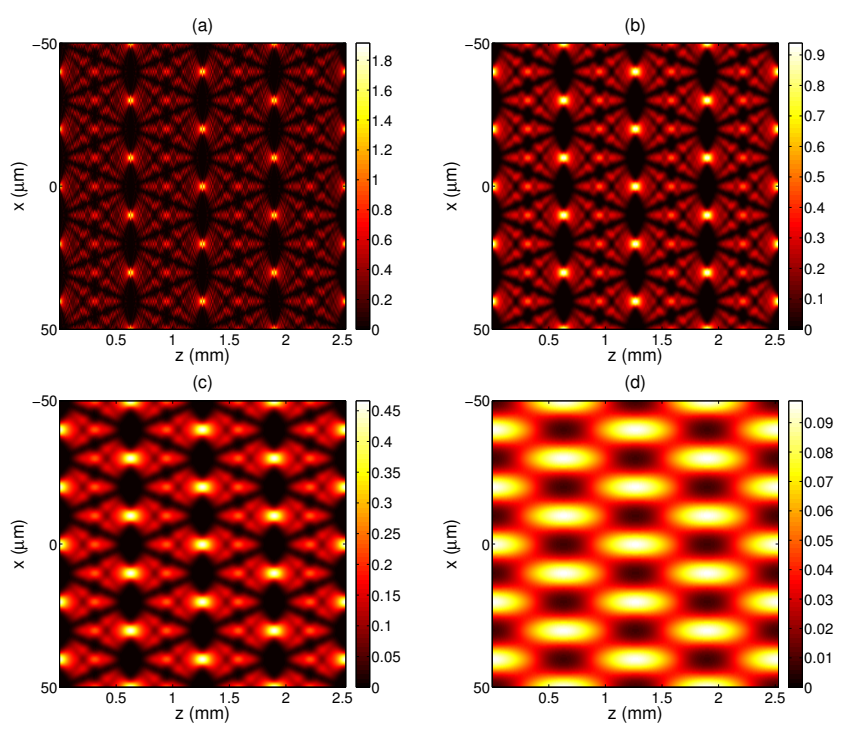

Fig. 4. Analytical self-images calculated using Eq. (9) with a Ronchi grating of period, $p=20 \mu \mathrm{m}$, nominal fill factor $\tau=$ $0.2 p$, and illuminated with a plane wave of wavelength, $\lambda=$ $632.8 \mathrm{~nm}$. Colormap corresponds to intensity. The amounts of randomness in the fill factor are: (a) $\omega=0$, (b) $\omega=p / 10$, (c) $\omega=p / 5$, and (d) $\omega=p / 2$. 


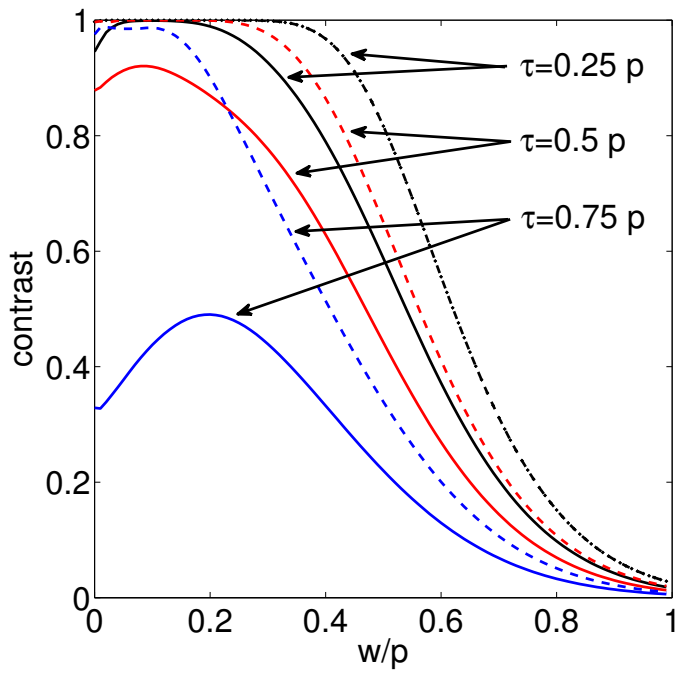

Fig. 5. Contrast of the first observable self-image in terms of randomness in the fill factor. The period of the grating is $p=20 \mu \mathrm{m}$ and three nominal fill factors. Analytical calculated by using Eq. (10) (solid line) and analytical calculated by using the classical definition of contrast, Eq. (11), (dashed line).

average far field intensity for different values of randomness in the fill factor. As can be observed, the effect of the randomness produces cancellation of higher diffraction orders. For completeness, we show in Figure 7 the behavior of the diffraction orders for different fill factors and fixed randomness, $w=p / 2$. Higher fill factors produce less powerful first diffraction order.

\section{NUMERICAL SIMULATIONS}

In this section, we perform a numerical validation of the obtained theoretical formalism by using a fast-Fourier transformbased direct integration method that uses the RayleighSommerfeld formula as propagation kernel [27]. We define the grating in a similar way as Figure 1. It is composed by slits periodically displaced and each slit has random fill factor around a nominal value that we establish as $\tau=p / 2$. In addition, we chose the random distribution Gaussian with null mean value without loss of generality. To understand the propagation process, we show in Figure 8 the near field intensity produced by a Ronchi diffraction grating with different amounts of randomness, (a) $\omega=0$, (b) $\omega=p / 10$, (c) $\omega=p / 5$, and (d) $\omega=p / 2$. The damage of the self-images is clearly observed. Anyway, since the theoretical intensity has been calculated as an average, we need to perform also an average on the numerical simulations for comparison. We show in Figure 9 the mean intensity corresponding to an ensemble of 1000 realizations of Figure 8. Finally, we calculate the numerical contrast in terms of the randomness of the fill factor by using Eq. 10, Figure 3 (dashdotted line). The similarity between analytical and numerical formalisms is clear, validating the obtained theoretical approach.

\section{CONCLUSIONS}

Manufacture errors in diffraction gratings have to be taken into account in many areas such as optical encoders or interferometry. In this work, we analyze the effect on the diffraction pattern of Ronchi gratings where the fill factor presents a certain random-

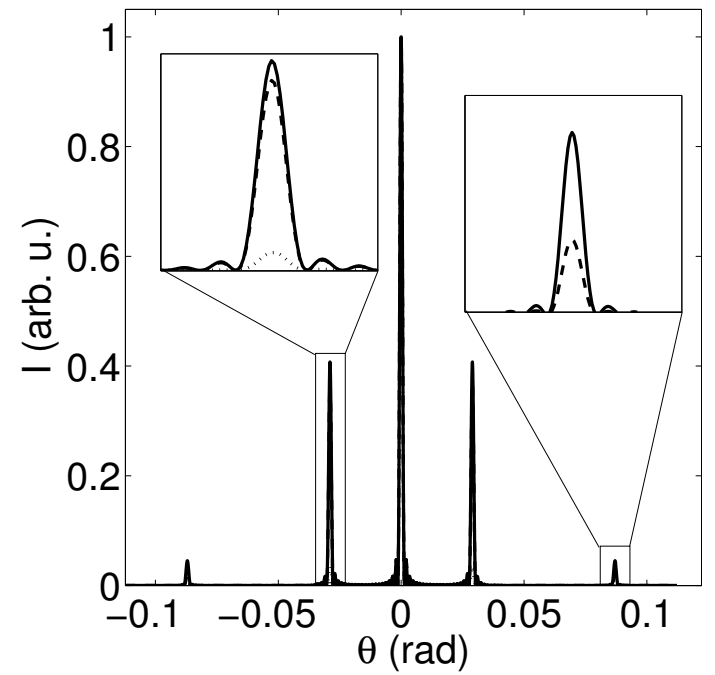

Fig. 6. Analytical far field mean intensity calculated by using Eq. (13). The period of the grating is $p=20 \mu \mathrm{m}$, the nominal fill factor is $\tau=p / 2$, and the amounts of randomness in the fill factor are: (solid line) $\omega=0$, (dashed line) $\omega=p / 10$, and (dot line) $\omega=p / 2$.

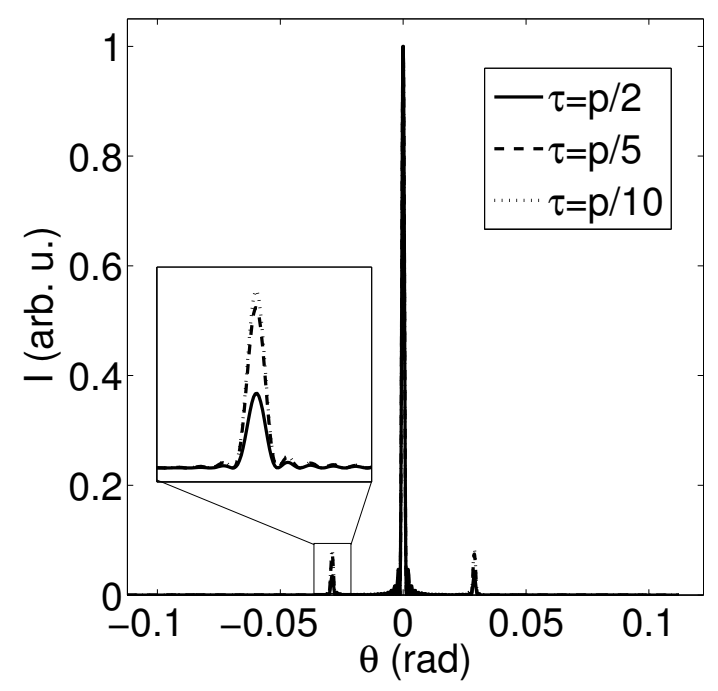

Fig. 7. Analytical far field mean intensity calculated by using Eq. (13). The period of the grating is $p=20 \mu \mathrm{m}$, the amount of randomness in the fill factor is $\omega=p / 2$, and the nominal fill factors are: (solid line) $\tau=p / 2$, (dashed line) $\tau=p / 5$, and (dot line) $\tau=p / 10$. 


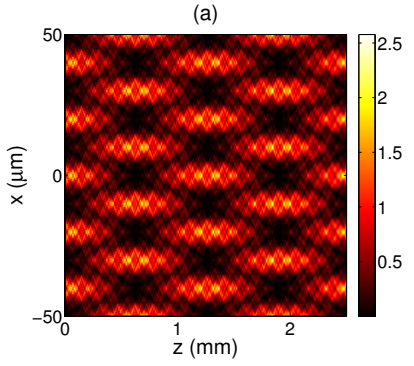

(c)

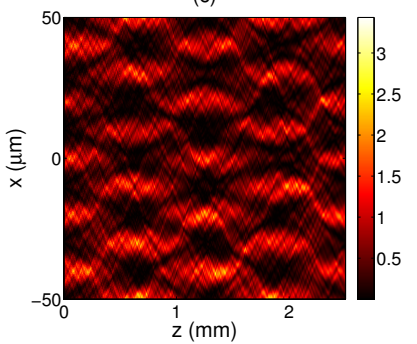

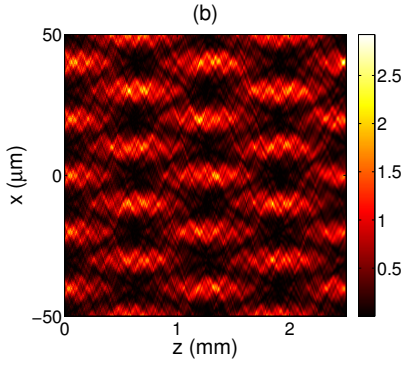

(d)

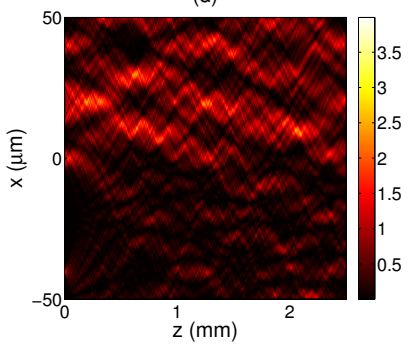

Fig. 8. Numerical self-images (one realization) with a Ronchi grating of period, $p=20 \mu \mathrm{m}$, nominal fill factor $\tau=p / 2$, and illuminated with a plane wave of wavelength, $\lambda=632.8$ $\mathrm{nm}$. Colormap corresponds to intensity. The amount of randomness in the fill factor are (a) $\omega=p / 10$, (b) $\omega=p / 5$, (c) $\omega=p / 2$, and (d) $\omega=p$.
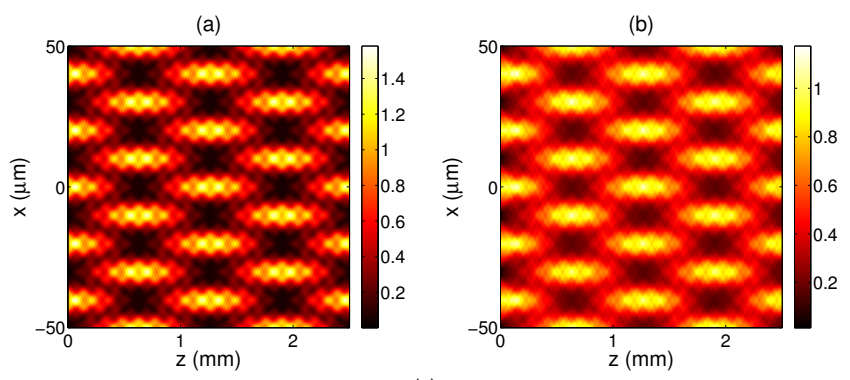

(c)

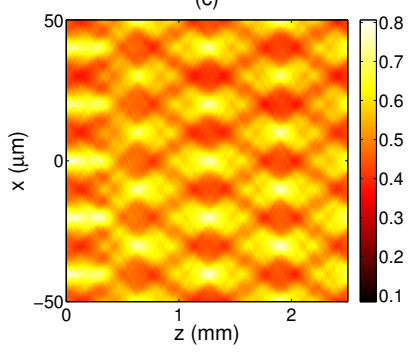

Fig. 9. Numerical self-images (average over 1000 realizations) with a Ronchi grating of period, $p=20 \mu \mathrm{m}$, nominal fill factor $\tau=p / 2$, and illuminated with a plane wave of wavelength, $\lambda=632.8 \mathrm{~nm}$. Colormap corresponds to intensity. The amounts of randomness in the fill factor are: (a) $\omega=p / 10$, (b) $\omega=p / 5$, and (c) $\omega=p / 2$. ness. This effect can be owed to etching processes or lithographic errors. We develop an analytical formulation and corroborate it with numerical simulations. The random fill factor produces a decreasing of the self-images contrast in terms of the amount of randomness and therefore, a cancellation of higher diffraction orders at the far field.

\section{FUNDING INFORMATION}

This work has been supported by project "Ecograb" RTC-20165277-5 of "Programa Estatal de Investigacion, Desarrollo e Innovacion Orientada a los Retos de la Sociedad" by Ministerio de Economia y Competitivdad and co-financed with structural funds of the European Union.

\section{REFERENCES}

1. A. Lohmann and D. Silva, "An interferometer based on the talbot effect," Optics Communications 2, 413-415 (1971).

2. G. S. Spagnolo, D. Ambrosini, and D. Paoletti, "Displacement measurement using the talbot effect with a ronchi grating," Journal of Optics A: Pure and Applied Optics 4, S376 (2002).

3. B. Oreb and R. Dorsch, "Profilometry by phase-shifted talbot images," Applied optics 33, 7955-7962 (1994).

4. S. Wei, S. Wu, I. Kao, and F. Chiang, "Measurement of wafer surface using shadow moiré technique with talbot effect," Journal of Electronic Packaging 120, 166-170 (1998).

5. C. A. Palmer and E. G. Loewen, Diffraction grating handbook (Newport Corporation Springfield, Ohio, USA, 2005).

6. L. M. Sanchez-Brea, F. J. Torcal-Milla, and E. Bernabeu, "Far field of gratings with rough strips," JOSA A 25, 828-833 (2008).

7. F. J. Torcal-Milla, L. M. Sanchez-Brea, and E. Bernabeu, "Self-imaging of gratings with rough strips," JOSA A 25, 2390-2394 (2008).

8. F. J. Torcal-Milla, L. M. Sanchez-Brea, and E. Bernabeu, "Double grating systems with one steel tape grating," Optics Communications 281, 56475652 (2008)

9. F. J. Salgado-Remacha, L. M. Sanchez-Brea, and E. Bernabeu, "Effect of fill-factor on the talbot effect of diffraction gratings," Journal of the European Optical Society-Rapid publications 6 (2011).

10. M. J. Madou, Manufacturing techniques for microfabrication and nanotechnology, vol. 2 (CRC Press, 2011).

11. F. J. Salgado-Remacha, F. J. Torcal-Milla, L. M. Sanchez-Brea, and E. Bernabeu, "Use of steel substrates in diffractive optics: Near field of high surface quality steel tape gratings," Optics and Lasers in Engineering 49, 356-360 (2011).

12. F. J. Torcal-Milla, L. M. Sanchez-Brea, and E. Bernabeu, "Diffraction of gratings with rough edges," Optics express 16, 19757-19769 (2008).

13. J. M. Rico-García and L. M. Sanchez-Brea, "Binary gratings with random heights," Applied optics 48, 3062-3069 (2009).

14. F. J. Torcal-Milla, L. M. Sanchez-Brea, and E. Bernabeu, "Talbot effect with rough reflection gratings," Applied optics 46, 3668-3673 (2007).

15. L. M. Sanchez-Brea, F. J. Torcal-Milla, and E. Bernabeu, "Talbot effect in metallic gratings under gaussian illumination," Optics communications 278, 23-27 (2007).

16. L. M. Sanchez-Brea and F. J. Torcal-Milla, "Near-field diffraction of gratings with surface defects," Applied optics 49, 2190-2197 (2010).

17. Y. Lu, C. Zhou, and H. Luo, "Talbot effect of a grating with different kinds of flaws," JOSA A 22, 2662-2667 (2005).

18. A. Lin and J. Phillips, "Optimization of random diffraction gratings in thin-film solar cells using genetic algorithms," Solar Energy Materials and Solar Cells 92, 1689-1696 (2008).

19. F. J. Torcal-Milla and L. M. Sanchez-Brea, "Diffraction by random ronchi gratings," Applied Optics 55, 5855-5859 (2016).

20. H. F. Talbot, "Lxxvi. facts relating to optical science. no. iv," The London and Edinburgh Philosophical Magazine and Journal of Science 9, 401407 (1836).

21. K. Patorski, "I the self-imaging phenomenon and its applications," Progress in optics 27, 1-108 (1989). 
22. E. Keren and O. Kafri, "Diffraction effects in moiré deflectometry," JOSA A 2, 111-120 (1985).

23. K. Engelhardt and P. Seitz, "High-resolution optical position encoder with large mounting tolerances," Applied optics 36, 2912-2916 (1997).

24. C.-F. Kao and M.-H. Lu, "Optical encoder based on the fractional talbot effect," Optics communications 250, 16-23 (2005).

25. J. Goodman, "Statistical optics," (2000).

26. F. J. Torcal-Milla, L. M. Sanchez-Brea, and J. Vargas, "Effect of aberrations on the self-imaging phenomenon," Journal of Lightwave Technology 29, 1051-1057 (2011).

27. F. Shen and A. Wang, "Fast-fourier-transform based numerical integration method for the rayleigh-sommerfeld diffraction formula," Applied optics 45, 1102-1110 (2006). 